Journal of medical \& pharmaceutical Sciences

Volume (5), Issue (3) : 30 Sep 2021

P: 69 - 83
AJSRP

ISSN: 2522-333X
مجلة العلوم الطبية والصيدلانية

المجلد (5)، العدد (3) : 30 سبتمبر 2021 م

ص: 69 - 83

\title{
Evaluation of patient satisfaction and the color difference between natural teeth and all-ceramic crowns fabricated from "Celtra press" treated with different surface finishing protocols. (Randomized controlled clinical trial)
}

\author{
Nourhan Sayed Saleh Ahmed \\ Mohamed Labib Zamzam \\ Maha Taymour \\ Karim Abo Bakr Mohamed \\ Faculty of Dentistry || Cairo University || Egypt
}

\begin{abstract}
All studies agree that glazing can produce a smooth porcelain surface, but although the glaze is an important factor in esthetics, with respect to light reflection, it can frequently change the color value of the restorations, reflecting more light than the natural teeth, thus creating an artificial effect on these restorations. A number of more recent studies have suggested that a polished surface may be as acceptable as a glazed surface. Many ceramists prefer polishing instead of glazing to control the surface luster. Other studies have reported no significant differences between polished and glazed surfaces.Since studies addressing the effect of surface finishing protocols on the color of ZLS ceramics are rare, thus our study is important to investigate this issue.

Methodology: 20 teeth esthetic zone requiring full - coverage crown -with their contralateral are sound and not severely discolored - were prepared to receive an all -ceramic crown. The crowns are divided into two groups: Group I: Teeth received crowns fabricated from glazed Celtra Press ceramic Group II: Teeth received crowns fabricated from polished Celtra Press. The color difference $\Delta \mathrm{E}$ was measured using an intraoral spectrophotometer and evaluated for each group. Shade matching to the contralateral tooth was evaluated by 2 experienced prosthodontists according (USPHS) criteria. Patient satisfaction of the two groups assessed using the (VAS) scale which is Binary and documented in the chart.

Results: Patient satisfaction was graded according to the (VAS) scale. There was no statistically significant difference between (Group I) and (Group II). The color change was recorded according to the $(\Delta \mathrm{E})$, the polished Celtra Press group recorded statistically non-significant higher color changes than the glazed Celtra Press group. shade matching was graded according to USPHS, where in Glazed Celtra Press group most of the patients stated Alpha score (80\%) while minority showed Bravo score (20\%). In the Polished Celtra Press group, all of the patients informed Bravo score $100 \%$.

Conclusion: Within the limitations of this clinical study:

Glazed and polished Celtra Press full coverage restorations revealed excellent patient satisfaction. Glazed celtra restorations have a clinically more acceptable shade matching.
\end{abstract}

Keywords: celtra, celtra press, glazing, polishing, ceramics, esthetics. 


\section{تقييم رضا المريض وفرق اللون بين الأسنان الطبيعية والتيجان الخزفية بالكامل المصنعة من "مطبعة سيلترا" المعالجة ببروتوكولات تشطيب الأسطح المختلفة:

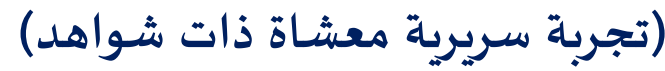 \\ كلية طب الفم والأسنان || جامعة القاهرة || مصر معر}

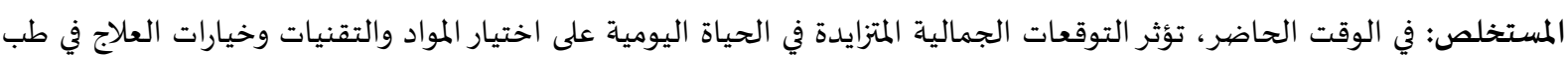

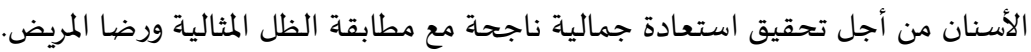

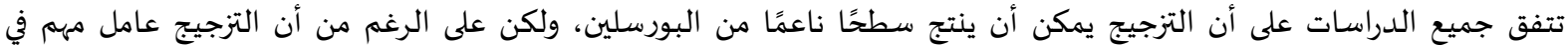

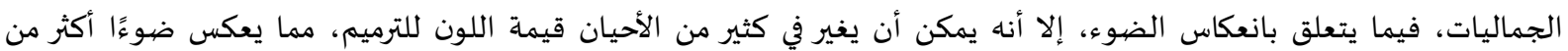

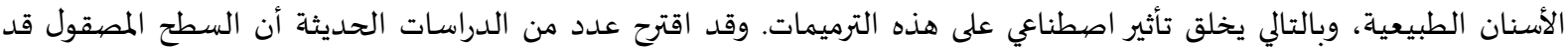

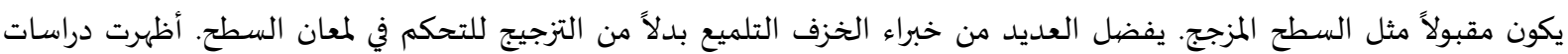

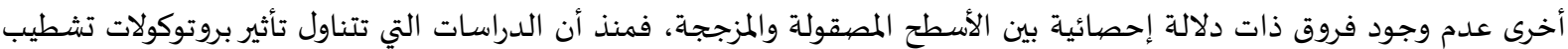

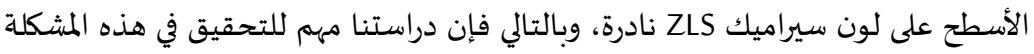
تم تصنيع 20 من التيجان الكاملة التغطية الخزفية (عشرة في كل مجموعة) للأسنان التي تتطلب ترميمات تغطية كاملة في المنطقة

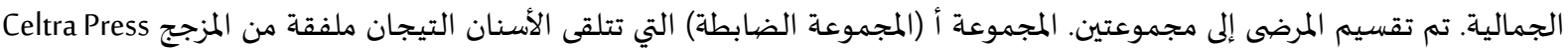
الخزفي والمجموعة B (مجموعة التدخل) الأسنان التي تستقبل تيجان مصنّعة من مصقول (Celtra Press) Dentsply Sirona). تم قياس فرق اللون E) (عتبة الإدراك الحسي) باستخدام مقياس الطيف داخل الفم وتم تقييمه لكل مجموعة تم تقييم الظل المطابق المعان

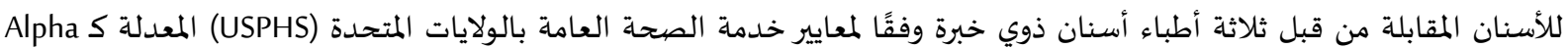

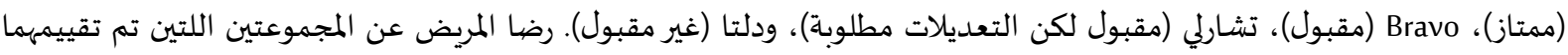

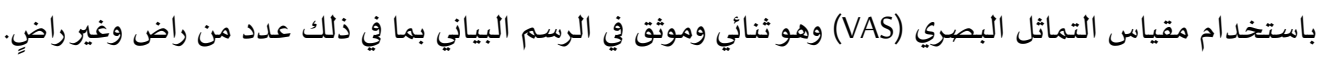

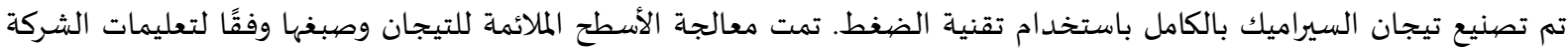

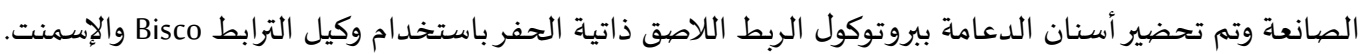
تم تصنيف رضا المريض وفقًا لمقياس التماثل البصري (VAS). لم يكن هناك فرق ذي دلالة إحصائية بين (المجموعة الأولى) و(المجموعة المانة

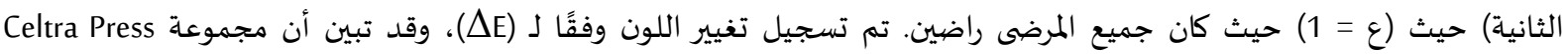

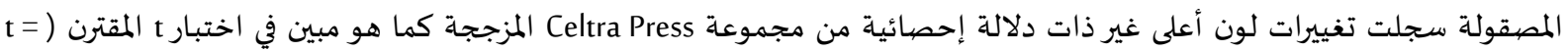

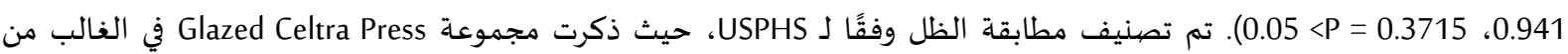
المرضى أن درجة ألفا (80\%) بينما أظهرت الأقلية درجة برافو (20\%) بدون أي سجل لدرجات تشارلي ودلتا (0).

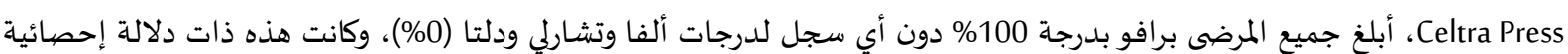

كما ثبت من اختبار تشي مربع (0.05>P = 0.0014)

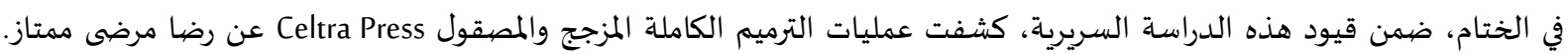

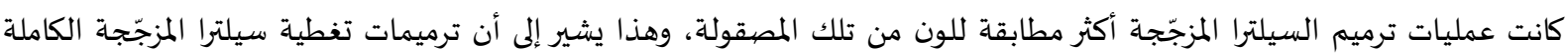
ذات غطاء ظاهري أكثر توافقًا من الناحية السريرية ويمكن التوصية باستخدامها كترميمات كايمات تغطية كاملة في المواقف السريرية للحصول على أفضل نتيجة جمالية.

الكلمات المفتاحية: سيلترا، مطبعة سيلترا، تلميع، سيراميك، تجميل. 


\section{1- Introduction}

The use of all - ceramic restorations has increased in the past several years mostly due to esthetic demands by patients. A number of ceramic materials have been developed for their esthetic capability, biocompatibility, color stability, and low thermal conductivity (Pires L.A. 2017) .

Dental ceramics are classified into: Glass-matrix ceramics, Polycrystalline ceramics, and resinbased ceramics. Evolution in glass- ceramics leads to the development of new zirconia reinforced lithium silicate ceramics that is composed of lithium silicate crystallites and $10 \%$ zirconia embedded in a glassy matrix. (Caner Yılmaz, (2008).

One of ZLS classes, celtra press (Dentsply Sirona) is a new material that can deliver the highest level of esthetics mimicking natural teeth based on having an amazing chameleon effect, perfect balance of translucency, and natural like opalescence only by polishing the material after pressing it (Ali Riza Tuncdemir, (2012).

After the pressing procedure, proper finishing and polishing of dental restorations are important in enhancing both the esthetics and longevity of restored teeth. Residual surface roughness, associated with improper finishing and polishing of dental restorations can result in a number of clinical difficulties for both the dentist and the patient. These problems include excessive plaque accumulation, gingival irritation, increased surface staining, and poor or suboptimal esthetics of the restored teeth, there have been many recommended techniques to decrease surface roughness, including auto glazing, super glazing, and polishing (Caner Yılmaz, 2008).

\section{Statement of problem}

After finishing the pressing procedure, the restoration will have a rough surface that can lead to loss of esthetic quality, thus it needs to be smoothened not only for the esthetics but also for biological reasons, this is done by glazing or polishing. The efficacy of the ceramic polishing systems is a controversial issue in literature. Several studies reported that the final surfaces obtained with the polishing procedure are not comparable with the final glazing surfaces. On the other hand, other authors have reported that the polishing systems exhibited the capacity of reaching surfaces similarly to those obtained after the glazing procedure and can produce a smooth surface that can be more esthetically similar to natural enamel.

Many authorities favor polishing given that a higher level of control is possible over final surface finish and that an added firing can add problems and time to the delivery Appointment-

\section{Materials and Methods}

A total of 20 patients were selected for the study with an age range between 20 to 60 years old. Each participant received an all-ceramic full coverage restoration for an upper tooth in the esthetic zone 
(central incisor- second premolar). Their chief complaint was to enhance their smile. The treatment plan was explained for each patient. Then, they agreed to sign the informed consent before proceeding to clinical work. They were able and willing to maintain good oral hygiene measures.

\section{3- Sample grouping}

A total of 20 all-ceramic crowns were divided into two main groups ( $n=10$ each) according to the material used for crown fabrication.

Group (I): 10 patients each with a single tooth receiving full coverage Glazed celtra press crown in esthetic area.

Group (II): 10 patients each with a single tooth receiving full coverage Polished celtra press crown in esthetic area.

\section{4.- Diagnostic phase}

\subsection{Intraoral examination}

Dental examination, periodontal evaluation, symmetry of gingival level, oral hygiene, dental caries, and para-functional habits were assessed.

TMJ evaluation was conducted and intra-oral examination revealed the presence of opposite occluding dentition in the area intended for restoration (Figure: 1).

\subsection{Photographs}

Pre-operative photographs for each patient were taken using $105 \mathrm{~mm}$ Nikon macro lens with a twin flash R1C1 mounted on Nikon D7100 DSLR camera.

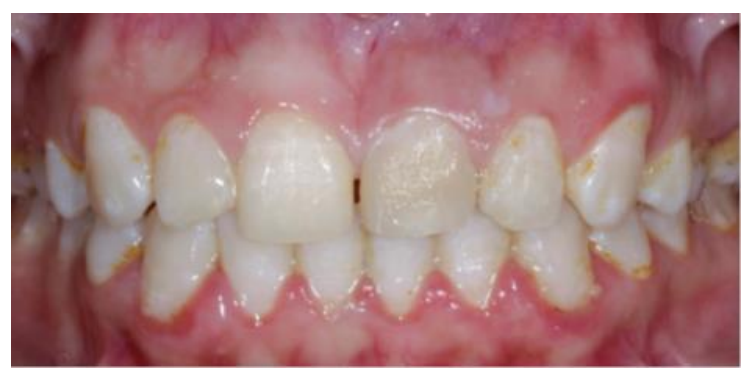

Figure (1) Intra-oral retracted photo

\subsection{Diagnostic casts}

Alginate impressions for upper and lower arches were taken using stock trays, poured with type IV dental stone. 


\subsection{Scaling and polishing}

Scaling and polishing were performed for each patient before shade selection in order to remove any dental plaque, calculus, and staining which will affect the accurate shade selection

\subsection{Shade selection}

The color of the tooth was recorded visually using a classical shade guide in accordance with the contra-lateral tooth under different light conditions: natural daylight and incandescent light to avoid metamerism with the help of two prosthodontists that performed Ishihara's test to determine color deficiency. Their results showed no color blindness.

Shade was confirmed with Vita Easy shade spectrophotometer. It was calibrated before starting each measurement. The spectrophotometer consisted of a base unit and a hand piece with a 5-mm diameter probe tip. The tooth was illuminated with spectrophotometer light, and reflected light was analyzed by the spectrophotometer (Figure: 2).

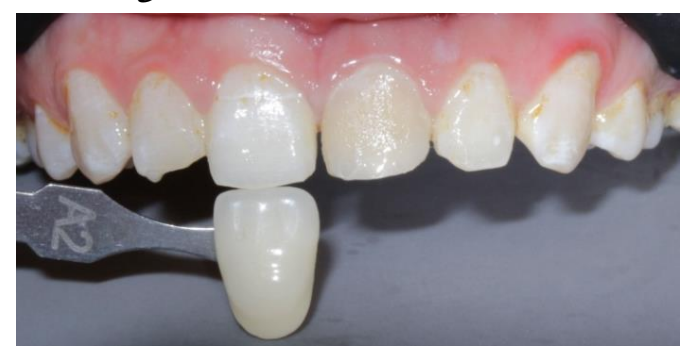

Figure (2): Shade selection with a classical shade guide.

\section{Tooth preparation}

\subsection{Silicon matrix index construction}

Putty silicon was used to obtain an intraoral index for each patient using condensation silicon impression material. Each index was vertically cut at the mid of the tooth requiring full-coverage restoration to assess the amount of preparation of incisal, labial, and palatal surfaces respectively. Another putty silicon index was fabricated directly inside the patient mouth which was used later for temporization

\subsection{Incisal preparation}

Vertical orientation grooves were done on the incisal edge of the tooth ensuring not to penetrate more than the diameter of the stone visually. The tapered diamond stone with a round end was placed parallel to the incisal edge to remove the projection between grooves resulting in $1.5 \mathrm{~mm}$ incisal surface preparation. 


\subsection{Labial preparation:}

The labial reduction was started with horizontal orientation grooves using depth cutter wheels in order to ensure even preparation thickness. Then the remaining island of the enamel was removed till the depth of original grooves to uniformly reduce the labial surface using a tapered diamond stone with a round end of $2.0 \mathrm{~mm}$ diameter

The preparation was carried out in two different planes following the contour of the labial surface. Then the preparation was verified with the silicon index to check the amount of labial preparation, it should be $1.5 \mathrm{~mm}$ on the incisal two- thirds and $1 \mathrm{~mm}$ on the cervical third according to the manufacturer's instruction.

\subsection{Proximal preparation}

A fine tapered diamond stone was used to slice and open the interproximal contact. Then a tapered diamond stone with a round end was used to create a chamfer finish line of $1.0 \mathrm{~mm}$ diameter.

\subsection{Cervical finish line}

The chamfer finish line was created supra-gingivally along the free gingival margin. The margin of the preparation was ended by a chamfer finish line $1.0 \mathrm{~mm}$ diameter using a tapered diamond stone with a round end (Figure 3).

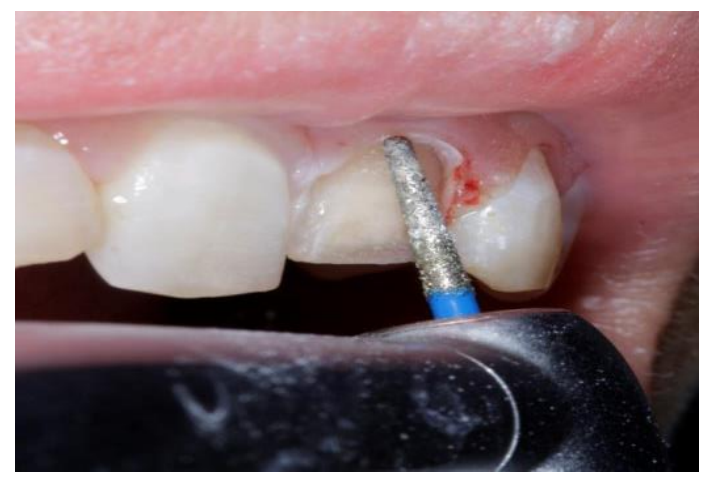

Figure (3): Deep chamfher finish line

\subsection{Palatal preparation}

The palatal fossa reduction was performed with a diamond football stone and the cingulum was prepared parallel to the long axis of the tooth with a tapered diamond stone with a round end of $1.0 \mathrm{~mm}$ diameter.

Then the preparation was verified with the silicon index to check the amount of palatal preparation ( $1.5 \mathrm{~mm}$ on palatal fossa and $1 \mathrm{~mm}$ in cingulum) 


\subsection{Finishing the preparation}

All sharp line angles, which might serve as a point for stress concentration, were rounded using finishing stone.

\section{The impression making}

The final impression was taken using vinyl polysiloxane addition silicon• in metal stock trays. Two - step impression techniques was performed, first putty viscosity was taken, the preparation was finished before applying the light body then light viscosity was applied by using an automatic mixing tip and dispensing impression gun which produced a complete homogenous mix.

\section{Provisionalization}

The direct fabricated silicon index was used for provisional restoration construction. The index was filled with temporary crown material and placed on the lubricated teeth of the patients intra-orally. After the complete setting of temporary material, the silicon index was removed. Then the temporary restoration was removed for proper finishing and polishing, followed by temporary cementation using non-eugenol acrylic-urethane polymer- based temporary cement.

\section{Master cast construction}

Once the dental laboratory received the final impression, master casts were poured with a type IV dental stone according to the manufacturer's instruction, with respect to water/powder ratio and mixing time. Vacuum mixing was used to ensure proper mixing and production of void - free casts. Dowel pin and die sawing were performed to allow proper designing of the final restoration.

\section{Scanning master cast:}

An extraoral scanner was used to scan the master cast to acquire a three-dimensional image for the abutment tooth on the computer screen in order to construct CAD/CAM milled castable Polymethyl methacrylate (PMMA) for the try- in the stage.

\section{Designing:}

Using Exocad software a virtual model was calculated from the scanned pictures and an automatic margin finder was used for preparation margin detection

\section{Try in stage}

The try-in was performed by using the CAD/CAM milled cast able Polymethyl methacrylate (PMMA) with the final shape of the crown. All prepared tooth surfaces were cleaned with the polishing brush then washed with water. The CAD/CAM PMMA was initially tried to check marginal fit, shape, contacts, contour, and then the overall integration with the lips and finally with the face. 


\section{Celtra press crown fabrication}

Celtra press ceramics are supplied in the form of ingots to be pressed into full contoured crowns

\section{Staining, glazing, and polishing stage}

\section{Esthetic try-in of all-ceramic crown}

Esthetic try-in of the all-ceramic crown was performed using a water-soluble gel• for proper checking of marginal fit, final contour, contacts, and color under natural day-light and confirmed with the Vita Easy-shade V.

\section{Bonding of all-ceramic crown}

- Crown surface preparation

The internal surface of the celtra press crowns was etched for 20 seconds with $9 \%$ hydrofluoric acid. The crown was rinsed with water then air dried by using three-way syringe. A single coat of silane coupling agent was then applied to the bonding surface of the crowns and left for 1 minute then air dried

- Tooth surface preparation

In order to remove remnants of provisional cements that may cause a significant decrease in the bond strength of the luting agent, a prophylaxis paste and polishing brush mounted in low speed contra angle was used for cleaning the tooth surfaces prior to bond in. Then, isolation was granted through the use of rubber dam

- Bonding

Finally, all teeth surfaces were dried gently for 5 seconds. Luting resin cement* was applied to the fitting surfaces of the crown using an automixing tip. The crown was placed to the tooth in position till complete seating using finger pressure. Excess cement was removed using sharp explorer after 2 seconds of preliminary light polymerization and the crown was then light polymerized for 40 seconds from each aspect of the tooth. A waxed dental floss was used inter-dentally for complete removal of excess cement in between crown and adjacent teeth. Moreover, an articulating paper was used to check for any occlusal interferences after complete curing and here in Figure (4) shows the crown after cementation.

\section{Postoperative instruction and care}

The patients were instructed to perform brushing and flossing regularly, using non-abrasive fluoridated toothpaste

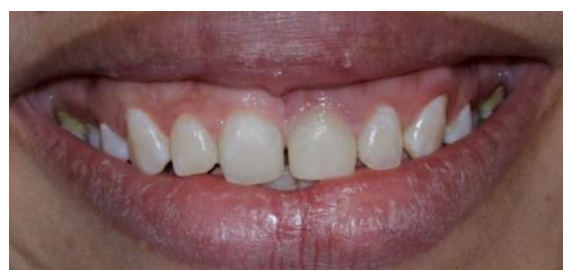

Figure (4): After cementation of the crown 


\section{Results}

\section{Statistical methods:}

All Data was collected, checked, revised, tabulated and entered into the computer. They were analyzed using IBM SPSS advanced statistics (Statistical Package for Social Sciences), version 20 (SPSS Inc., Chicago, IL). Categorical data were described as numbers and percentages. Comparisons between categorical variables will be performed using the chi square test or fisher exact as appropriate. A p-value less than or equal to 0.05 was considered statistically significant. All tests were two tailed

\subsection{Color changes}

Descriptive statistics showing mean values and standard deviation of color change test results measured in $(\Delta \mathrm{E})$ for both groups.

The mean \pm SD values of color changes recorded for the polished Celtra Press group were $(1.15 \pm 0.53 \Delta \mathrm{E})$ meanwhile the mean $\pm \mathrm{SD}$ value recorded with glazed Celtra Press group $(0.83 \pm 0.47 \Delta \mathrm{E})$.

It was found that the polished Celtra Press group recorded statistically non-significant higher color changes than the glazed Celtra Press group as indicated by paired t-test $(t=0.941, P=0.3715>0.05)$.

\begin{tabular}{|l|l|c|c|c|c|c|c|}
\hline \multirow{2}{*}{ Variables } & \multirow{2}{*}{ Mean } & \multirow{2}{*}{ SD } & & 95\% CI & \multicolumn{2}{l|}{ Range } \\
\cline { 5 - 9 } & & & & Lower & Upper & Mini. & Maxi. \\
\hline \multirow{2}{*}{ Material group } & Glazed Celtra Press & 0.83 & 0.47 & 0.44 & 1.22 & 0.40 & 1.70 \\
\cline { 2 - 8 } & PolishedCeltra Press & 1.15 & 0.53 & 0.68 & 1.62 & 0.40 & 2.50 \\
\hline \multirow{2}{*}{ t-test } & t-value & 0.941 & & & & & \\
\cline { 2 - 8 } & P value & $0.3715 \mathrm{~ns}$ &
\end{tabular}

\subsection{Shade match}

Shade match in relation to esthetics associated with glazed and polished Celtra Press restorations.

In the glazed celtra Press group predominantly of the patients stated Alpha score (80\%)while the minority showed Bravoscore(20\%)with no record for Charlie and Delta scores (0\%). In the Polished Celtra Press group, all of the patients informed Bravo score $100 \%$ with no record for Alpha, Charlie, and Delta scores $(0 \%)$. This was statistically significant as proved by chi - square test $(p=0.0014<0.05)$

\begin{tabular}{|l|l|c|c|c|c|}
\hline \multirow{2}{*}{ Variable } & Parameter & \multicolumn{4}{|c|}{ Shade match } \\
\cline { 2 - 6 } & Outcome & Alpha & Bravo & Charlie & Delta \\
\hline \multirow{2}{*}{ Material group } & Glazed Celtra Press & $8(80 \%)$ & $2(20 \%)$ & $0(0 \%)$ & $0(0 \%)$ \\
\cline { 2 - 6 } & Polished Celtra Press & $0(0 \%)$ & $10(100 \%)$ & $0(0 \%)$ & $0(0 \%)$ \\
\hline \multirow{2}{*}{ Chi square test } & Chi value & 10.2 & $0.0014 *$ \\
\cline { 2 - 6 } & P value & \multicolumn{4}{|l}{} \\
\hline
\end{tabular}




\subsection{Patient satisfaction}

Patient's satisfaction in relation to aesthetics associated with glazed and polished Celtra Press restoration groups.

All the patients (100\%) were satisfied while none was $(0 \%)$ dissatisfied by the restoration in both groups. This was statistically non-significant as proven by chi square test $(p=1>0.05)$

\begin{tabular}{|l|l|c|c|}
\hline \multirow{2}{*}{ Parameter } & \multicolumn{2}{|c|}{ Patient satisfaction } \\
\cline { 2 - 4 } & Outcome & Satisfied & Dissatisfied \\
\hline \multirow{2}{*}{ Material group } & Glazed Celtra Press & $10(100 \%)$ & $0(0 \%)$ \\
\cline { 2 - 4 } & PolishedCeltra Press & $10(100 \%)$ & $0(0 \%)$ \\
\hline \multirow{2}{*}{ Chi square test } & Chi value & \multicolumn{2}{|c|}{0} \\
\cline { 2 - 4 } & P value & \multicolumn{2}{|c|}{$1 \mathrm{~ns}$} \\
\hline
\end{tabular}

\section{Discussion}

This study was a randomized, double - blinded clinical trial where randomization was carried out by using computerized Sequence generation to eliminate the risk of selection bias of the included patients.

Since the main investigator performed all the procedures during this study, then, the evaluation of the outcome had to be performed by experienced evaluators who were blinded and did not know the patients belong to which group.

Visual shade matching using shade guides is still the most commonly used method due to its simplicity in comparing and matching the color of shade tabs with the color of teeth, Classical shade guide was used in this study due to its extensive use in dental clinics and several studies recommended that the color of the shade tabs are evenly distributed and the shade guide enables accurate shade matching with the natural dentition. This was in accordance with ((Vidhya Parameswaran et al, 2016) who stated that the visual method producing more accurate results than the spectrophotometric method, and VITAPAN Classical shade guide was more accurate than the spectrophotometer (CorcodelN., (2012).

However visual shade matching is considered as a skill acquired clinical experience and color education and training are considered very important for accurate shade matching. Therefore, in order to standardize the shade selection process, two prosthodontists were used as evaluators of the shade to the contra-lateral. All of them have undergone Ishihara's test to determine color deficiency. Their results showed no color blindness. This was in accordance with ((Paravina, 2000) and((Clary et al., 2016) which concluded that education and training resulted in an improvement of shade matching results as compared to the results before training.

Since the consistency and reliability of visual shade matching are questioned, the spectrophotometer (Vita Easyshade) was used as the main tool in this study to detect the color of the natural teeth as Chen et al., 2012 performed a systematic review of visual and instrumental 
measurements for tooth shade matching and concluded that instrumental method provides the most precise and accurate shade-matching outcomes.

Also, (Bahannan et al, 2014) and (Alshiddi et al, 2015) stated that matching the shade of natural teeth using a spectrophotometer is more accurate than a conventional method using a shade guide.

All teeth included in this study were anterior teeth, first and second premolars. This is due to their presence in the esthetic zone, the most visible teeth in the dental arch where esthetics, shade, and patient satisfaction play an important role for successful restoration.

In this study, the ceramic restorations were fabricated from the same dental ceramic material "Celtra press (Dentsply sirona)" which is zirconia-reinforced lithium silicate ceramic (ZLS).

Celtra Press is a multiphase ceramic consisting of a glass matrix and lithium disilicate crystals having a crystal length of about $1.5 \mu \mathrm{m}$ plus nano-scale lithium phosphate. In addition to $\mathrm{Li} 2 \mathrm{O}$ and $\mathrm{SiO} 2$, Celtra Press contains about $10 \%$ zirconia ( $\mathrm{ZrO} 2)$.

Conventional all - ceramic full coverage preparation was performed by preparing the tooth according to manufacturer's guidelines for all-ceramic crown with smooth, round contours and angles, supra-gingival deep chamfer finish line $1 \mathrm{~mm}$ deep with round internal angles, incisal/occlusal reduction of $1.5 \mathrm{~mm}$, and labial reduction performed in 2 planes.

In order to standardize our preparation design, two-wheel depth cutter was used to have optimum depth cutting. Then, a silicon index was performed for checking the final preparation depth and design. This procedure ensured uniform reduction.

The final impression was taken with the addition silicon impression material since it has low dimensional change, relatively short setting time, and with moderate to high tear resistance. As there are no by products to the polymerization reaction, impressions are dimensionally stable and can be poured at the convenience of the operator.

Provisionalization was an integral part of the treatment process to imagine the final shape, protect teeth against sensitivity (if vital), more comfortable for the patient, and high esthetic appearance although a short time was required from the preparation procedure to bonding. The provisional restorations were temporary cemented using eugenol-free temporarily cement.

Pressing techniques were used in this study for both materials since it is very popular in the laboratory due to ease of use and the resultant restoration is of high fracture toughness and excellent marginal fit.

In the present study, according to ((Magne et al., 2005) remnants of provisional cement were removed by a Prophylaxis paste and polishing brush mounted in low -speed contra - angle to ensure that the tooth surface is completely cleaned from provisional cement and contaminations which might affect the bond strength. 
The use of clear glycerin water - soluble gel as an optical fluid to help predict the influence of resin cement shade on the definitive restoration $=$

Self - adhesive resin cement was used for the final cementation of the ceramic restorations. It's dual- cured translucent resin cement. The resin cement was applied after surfaces of the all-ceramic crowns were etched with $9 \%$ hydrofluoric acid for 20 seconds, then a silane coupling agent was applied and air - dried for 60 seconds, then the cement was applied on the crown and the crown was seated with finger pressure and the excess cement was removed.

\section{Color change results:}

There was no statistically significant difference regarding $\Delta \mathrm{E}$ between Group $(\mathrm{A})$ the glazed celtra group where (the mean $\pm \mathrm{SD}=0.83 \pm 0.47 \Delta \mathrm{E}$ ) and Group $(\mathrm{B})$ the polished celtra group where (the mean \pm $\mathrm{SD}=1.15 \pm 0.53 \Delta \mathrm{E}$ ), This might be attributed to the fact that the two groups were pressed from the same ceramic material (Celtra press) and treated with different surface finishing protocols that are both recommended by the manufacturer as they claim that celtra is fast and easily polished and resulted in high surface gloss comparable to glazing due to its ultra-fine microstructure as it composed of a glass matrix and lithium silicate crystals having a crystal length of about 500-1400 nm plus nano-scale lithium phosphate and about $10 \%$ zirconia ( $\mathrm{ZrO} 2)$, which is dissolved completely in the glass phase.

This was in agreement with Duygu Sarac et al, 2006; who compared the effect of different surface finishing methods on the color and surface texture of a feldspathic ceramic and they concluded that the use of a polishing kit alone or preceding polishing paste or polishing stick application created surfaces as smooth as glazed specimens and the color differences of all groups were found to be at the acceptable level.

It is also related to the strict and meticulous shade matching protocol followed, as the shade was taken according to the classical shade guide and then confirmed with Vita Easyshade $V$ spectrophotometer. This was in accordance with Bagis and Turgut, 2013 who stated that to achieve a natural-looking restoration, two different steps need to be performed: select the best possible shade using a shade guide and/or an electronic shade-taking instrument, and reproduce this shade with an appropriate dental material.

Group (B) showed statistically non-significant higher color changes than group (A) but within the acceptable thresholds of non-perceivable color change reported in the literature. This could be explained by the change in brightness, represented in L* values which decreased with polishing, this could be attributed to changes in the amount of reflected light, but a more lustrous surface would reflect more light and appear luminous and of higher value (Wang, H. et al 2011).

This was in accordance with (Ali Riza Tuncdemir et al, 2012) who Evaluated the effects of different porcelain polishing techniques on the color change and surface roughness of feldspathic 
porcelains and lithium disilicate glass-ceramic system and they concluded that polishing procedures were not able to provide a porcelain surface as smooth as the glazed surface for the tested IPS empress II and Ceramco 3 porcelains. Among the polishing techniques, glazing was more successful than mechanical polishing for the surface color change of the porcelains. Later on Akar, et al 2014 stated that for all ceramic types, the smoothest surfaces were obtained after glazing, on the other hand After finishing with the polishing kit an acceptable surface roughness values were achieved.

Contradictory to these results, Mehmet Mustafa Özarslan et al 2016, evaluated surface roughness and color differences caused from different finishing procedures of VITA Enamic and they concluded using Technical polishig Kit instead of Glaze may cause better clinical performance for Vita Enamic restorations in the aspects of both surface roughness and shade matching.Shade matching results:

The statistically significant difference regarding to the USPHS scores between group (A) and group (B) $(p=0.0014<0.05)$ where in glazed celtra press group predominantly of the patients stated Alpha score (80\%) while minority showed Bravo score $(20 \%)$ whereas in polished celtra press group all of patients stated Bravo score $100 \%$ may be due to the dentists are well experienced and more sensitive with regard to identifying deviations in shade than are laypeople which resulted from the change in brightness, represented in $L^{*}$ values which decreased with polishing, this could be attributed to changes in the amount of reflected light, but a more lustrous surface would reflect more light and appear luminous and of higher value (Wang, H. et al 2011).

In 1994 Chung et al. Suggested that color difference was mainly determined by the lightness rather than the hue and chroma. These results are in agreement with Tuncedemir et al. 2013 who claimed that the higher color change observed with polished ceramics could be attributed to the rubber polishers that cause staining of the roughened ceramic surface.

And in 2016 Lee et al investigated the effects of surface finishing methods on the color distribution of colored anatomic-contour zirconia, they concluded that the color appearance of the colored zirconia crowns was strongly influenced by the surface-finishing methods

\section{Patient satisfaction results:}

Although group (B) has a higher non-significant difference than group (A) regarding the color change and there was a significant difference between the two groups regarding the USBHS score, all the patients were satisfied with their restorations. And there was no statistically significant difference between the two groups $(p=1>0.05)$. These results might be reasonable, since dentists are more sensitive with regard to identifying deviations in shade than are laypeople and it is the reflection of meticulous shade matching protocol followed. 
Earlier in 2002, Al-Wahadni et al, found that patients tended to rate restorations more favorably when the restoration was received in an academic institution, denoting that patients' pride in the school or positive relationship with the dental student may have elevated his or her opinion of the care received.

Contradicting to our results those by Shah et al., 2014 who found the overall rating of patient satisfaction was moderate. They explained that patient level of education might affected the results. Patients with secondary, post-secondary and tertiary level of education were more satisfied with tooth color compared to patients with primary level of education.

Finally the null hypothesis of this study was rejected as there were a significantdifference regarding the shade match between glazed and polished celtra press ceramics.

\section{References:}

- Alshiddi I.F. \& Richards L.C. (2015)"A comparison of conventional visual and spectrophotometric shade taking by trained and untrained dental students." Aust. Dent. Journal.; 60: 176-81.

- Ali Riza Tuncdemir, (2012) "The Effects of Porcelain Polishing Techniques on the Color and Surface Texture of Different Porcelain Systems" Materials Sciences and Applications Vol.3 No.5

- Bahannan S.A. (2014): "Shade matching quality among dental students using visual and instrumental methods." Journal of Dentistry.; 42: 48-52.

- Caner Yılmaz, (2008): "Stability of Glazed and Polished Dental Porcelains", Journal of Prosthodontics 17- 20-24

- Chen H., (2012): "A systematic review of visual and instrumental measurements for tooth shade matching". Quintessence International journal.; 43: 649-59.

- Clary J.A., (2016): "Influence of light source, polarization, education, and training on shade matching quality". Journal of Prosthetic Dentistry.; 116: 91-7.

- Corcodel N., (2012) "Evaluation of two different approaches to learning shade matching in dentistry". Acta Odontolgica. Scandinavica journal.; 70: 83-8.

- Chung, K. (1994): "Effects of finishing and polishing procedures on the surface texture of resin composites". Dent Mater. journal.325-330

- Magne P., (2005) "Immediate dentin sealing improves bond strength of indirect restorations". Journal of Prosthetic Dentistry. 94: 511-9.

- Paravina R.D. (2000). "Techniques for improvement of clinical shade matching procedures". Ph.D. dissertation, University of Nis School of Medicine, Nis, Serbia.

- Pires L.A. (2017). "Effects of the type and thickness of ceramic, substrate, and cement on the optical color of a lithium disilicate ceramic". Journal of Prosthetic. Dentistry. 117: 144-9.

- Shah R.J. (2014) "A Study of Patient Satisfaction with Maxillary Anterior Teeth Restorations and Desirable Esthetic Treatment Options". Journal of. Dent. Med. Sci.;13: 79-86 
- Vidhya Parameswaran. (2016). "Comparison of accuracies of an intraoral spectrophotometer and conventional visual method for shade matching using two shade guide systems" The Journal of Indian Prosthodontic Society, | Vol 16 | Issue 4 Oct-Dec.

- Wang, H. (2011) "Influence of varied surface texture of dentin porcelain on optical properties of porcelain specimens". Journal of Prosthetic Dentistry. 105 (4), 242-248. 